\title{
Clinical analysis of X-linked agammaglobulinaemia and common variable immunodeficiency in children - what should paediatricians know?
}

\author{
Małgorzata M. Pac', Ewa Bernatowska'1, Teresa Jackowska², Bożena Mikołuć ${ }^{3}$ \\ 'Department of Immunology, The Children's Memorial Health Institute, Warsaw, Poland \\ 2Department of Paediatrics, Medical Centre of Postgraduate Education, Warsaw, Poland \\ ${ }^{3}$ Department of Paediatrics, Rheumatology, Immunology, and Metabolic Bone Diseases, Medical University of Bialystok, \\ Bialystok, Poland
}

\section{ABSTRACT}

\begin{abstract}
Aim of the study: To present relevant clinical features of X-linked agammaglobulinaemia (XLA) and common variable immunodeficiency (CVID) in children, helpful in the everyday practice of paediatricians and general practitioners. To analyse the similarities and differences between XLA and CVID.

Material and methods: The retrospective analysis of the onset of symptoms, age of clinical diagnosis, basic immunological parameters, and infectious and non-infectious complications in 11 XLA and 21 CVID patients from a single centre was carried out.

Results: XLA boys presented the first symptoms in the second half of the first year of life. They had extremely low B-cell numbers (mean: 15 cells/ $\mu \mathrm{l}, 0.45 \%$ ), and immunoglobulin levels (median IgG: $0.38 \mathrm{~g} / \mathrm{l}$ ). Patients with CVID presented later occurrence of symptoms (median: two years), higher numbers of B cells (mean: 304 cells $/ \mu \mathrm{l}, 12.5 \%$ ), and higher immunoglobulin G levels (median: $2.92 \mathrm{~g} / \mathrm{l}$ ), but lower T CD4 cells. IgM and IgA levels were diminished in both groups, with lower values in XLA patients. Relevant differences $(p<0.05)$ in B-cell numbers and IgG levels between XLA and CVID groups were observed. Severe clinical complications such as chronic lung disease, bronchiectasis, autoimmune disorders, splenomegaly, and lymphadenopathy were more often observed in CVID.

Conclusions: An earlier onset of symptoms, extremely low B-cell numbers and immunoglobulin levels, and normal numbers of T cells point to XLA. Hypogammaglobulinaemia accompanied by non-infectious complications and diminished CD4 T cells are more suggestive of CVID. Greater awareness between paediatricians and general physicians should lead to a proper diagnosis, earlier treatment, avoidance of severe life-threatening complications, and better quality of life.
\end{abstract}

KEY WORDS:

immunoglobulins, autoimmunity, CVID, XLA.

\section{INTRODUCTION}

Primary antibody deficiencies (PADs) constitute the largest group of primary immune deficiencies (PIDs) and account for approximately $55 \%$ of all PIDs in Europe (www.esid.com) and up to $78 \%$ in the USA [1]. The spectrum of antibody disorders is very wide, ranging from markedly reduced immunoglobulin levels and ma-

\section{ADDRESS FOR CORRESPONDENCE:}

Małgorzata M. Pac, Department of Immunology, The Children's Memorial Health Institute,

20 Dzieci Polskich Ave, 04-730 Warsaw, Poland, ORCID: 0000-0003-2071-3297,

e-mail: malgorzata.pac@wp.pl 
ture B cells to selective antibody deficiency and normal numbers of B lymphocytes. Patients with severe antibody deficiencies usually present symptoms in the second half of the first year of life, until maternal antibodies have decreased below the protective level. Affected individuals share a clinical phenotype with recurrent or severe infections of the respiratory and gastrointestinal tract, chronic inflammation, and autoimmune phenomena. Encapsulated bacteria, such as Streptococcus pneumoniae or Haemophilus influenzae are the most common pathogens, while fungal and viral infections are hardly ever observed.

Among humoral defects, X-linked agammaglobulinaemia (XLA) and common variable immunodeficiency (CVID) are the most frequent symptomatic PADs. The incidence of CVID is estimated between $1: 20,000$ and $1: 200,000[1,2]$. Clinically it is a heterogeneous group of disorders, with the different onset of symptoms from childhood to adulthood, and a broad spectrum of symptoms including infections, autoimmunity, enteropathy, lymphoid malignancy, and granulomas. The pathogenesis of that syndrome is not clear, but either B-cell or T-cell differentiation failure is described as well as ineffective differentiation of B cells into memory cells and further into plasma cells [2-6]. According to ESID criteria (www. esid.org), CVID can be recognised in children over four years old, but earlier onset of symptoms may be observed. Although CVID is usually diagnosed in adulthood, variable proportions of children have such diagnosis $[7,8]$.

XLA presents one of the most frequent monogenic immune deficiencies with almost complete arrest of B-cell differentiation in bone marrow at pre-B-cell stage due to a defect in the Bruton's tyrosine kinase (Btk) gene. Following this defect in XLA patients a marked reduction of all classes of immunoglobulin and specific antibody response to immunisation is observed [9-11].

To prevent severe infections in the course of antibody deficiency life-long replacement immunoglobulin therapy (RIgT) is indicated for both groups [12-15]. Delayed diagnosis and inadequate treatment may lead to severe organ damage, worse quality of life, or even death of affected individuals. Awareness among paediatricians, general physicians, and nurse practitioners of PADs leads to proper diagnosis and treatment. Clinical and laboratory analyses of different types of PADs have been reported [13-18]. Less is known about the direct comparison of XLA and CVID in the paediatric population.

In this study, we present and compare clinical courses of XLA and CVID in children and point out the relevant features helpful for paediatricians to plan further diagnostics and start treatment.

\section{MATERIAL AND METHODS}

The study group included 11 boys with a definitive diagnosis of XLA and 21 children (17 boys and four girls) with a probable diagnosis of CVID according to
ESID criteria (www.esid.org.com), under the care of one of the largest centres for primary immunodeficiencies in children between 1990 and 2016. Clinical and laboratory data of patients were collected retrospectively from medical records. Serum immunoglobulin levels (G, A, M) were determined at diagnosis, according to generally accepted methods and compared to reference values for age-matched healthy groups. The percentage of peripheral blood lymphocyte subpopulations were determined by conventional three-colour direct immunofluorescence, with conjugated monoclonal antibodies (mAbs): CD4+/ CD8+/CD3+, and CD3+/CD56+/CD19+. Molecular analyses of XLA were done in all 11 boys courtesy of the Department of Immunology, Erasmus MC, Rotterdam. The results were evaluated using the Mann-Whitney two-sided rank U-test measured by means of Statistica for Windows release 5.0 (www.statsoft.com). The differences between the studied values were considered statistically significant at a $p$ level $<0.05$. The study was approved by the Bioethics Committee at CMHI, and written consent was received from the patients or their parents/guardians.

\section{RESULTS}

Table 1 presents clinical and demographic characteristics of XLA and CVID patients. The mean concentration of IgG before RIgT introduction was significantly lower $(p<0.05)$ in XLA patients compared to CVID ( $1.0 \mathrm{~g} / \mathrm{l} \mathrm{vs.}$ $3.2 \mathrm{~g} / \mathrm{l})$, and the median was even lower. Two other main immunoglobulin classes (IgA, IgM) were also low in both groups, with no statistical significance. As predicted, the number and percentage of B cells were substantially lower in XLA. An earlier mean onset of symptoms occurred in XLA, while more profound diagnosis delay concerned CVID. The family history was positive in $30 \%$ of XLA patients and sporadic in the CVID group. Neither malignancies nor deaths were reported in either studied group within the observation time.

The analysis of clinical symptoms and complications is presented in Table 2. The frequency of respiratory infections was similar in both groups. In CVID ear, nose, and throat (ENT) infections were observed in over 50\% of patients, while in XLA less frequently. Meningitis or sepsis were reported more often in CVID patients $(19 \%$ vs. $9 \%$ ). The frequency of bronchiectasis was comparable in both groups. Granuloma, interstitial pneumonia, or fibrosis was reported only in single CVID patients. One boy underwent lobectomy due to pulmonary fibrosis. Another one had granuloma in the liver and lungs. Gastrointestinal problems, including diarrhoea and inflammatory bowel disease, were more frequently diagnosed in XLA. Autoimmune cytopaenias concerned mainly CVID patients. Almost one third presented neutropaenia, thrombocytopaenia, and leucopaenia. In the XLA group neutropaenia was seen in $18 \%$. Over $40 \%$ of the CVID group 
TABLE 1. Clinical and demographic characteristics of X-linked agammaglobulinaemia and common variable immunodeficiency children at diagnosis

\begin{tabular}{|l|c|c|}
\hline Characteristics & $\begin{array}{c}\text { XLA } \\
(n=11)\end{array}$ & $\begin{array}{c}\text { CVID } \\
(n=21)\end{array}$ \\
\hline IgG median (g/l) & $0.38^{*}$ & 2.92 \\
Mean concentration (g/l) & $1.0^{*}$ & 3.1 \\
Range (g/l) & $0.0-4.03$ & $0.08-7.7$ \\
\hline IgA median (g/l) & 0.06 & 0.06 \\
Mean concentration (g/l) & 0.08 & 0.22 \\
Range (g/l) & $0.01-0.44$ & $0.01-1.21$ \\
\hline IgM (g/l) median & 0.24 & 0.39 \\
Mean concentration (g/l) & 0.19 & 0.65 \\
Range (g/l) & $0.02-0.68$ & $0.01-3.04$ \\
\hline Blymphocytes mean percentage $(\%)$ & $0.45^{*}$ & 12.5 \\
Range (\%) & $0.0-1.1$ & $0.3-28.7$ \\
Mean absolute number (cells/ $/ \mathrm{l})$ & $15^{*}$ & 304 \\
\hline Mean onset of symptoms & $1 \mathrm{y} 3 \mathrm{~m}$ & $2 \mathrm{y} 9 \mathrm{~m}$ \\
Median & $8.5 \mathrm{~m}$ & $2 \mathrm{y}$ \\
Range & $3-36 \mathrm{~m}$ & $1 \mathrm{~m}-6 \mathrm{y}$ \\
\hline Mean age of clinical diagnosis & $3 \mathrm{y} 8 \mathrm{~m}$ & $9 \mathrm{y} 7 \mathrm{~m}$ \\
Median & $3 \mathrm{y}$ & $9 \mathrm{y}$ \\
Range & $1-7.5 \mathrm{y}$ & $5-16 \mathrm{y}$ \\
\hline Diagnosis delay & $1 \mathrm{y} 6 \mathrm{~m}$ & $7 \mathrm{y} 2 \mathrm{~m}$ \\
\hline Family history & $30 \%$ & $9.5 \%$ \\
\hline Genetic confirmation & Yes & No \\
\hline Female/male & $0 / 11$ & $4 / 17$ \\
\hline
\end{tabular}

$X L A-X$-linked agammaglobulinaemia, CVID - common variable immunodeficiency, $y$ - years, $m-$ months, ${ }^{*} p<0.05$

TABLE 3. Phenotypic characteristics of basic lymphocyte subsets in $X$-linked agammaglobulinaemia and common variable immunodeficiency (mean values)

\begin{tabular}{|c|c|c|}
\hline Lymphocyte & $\begin{array}{c}\text { XLA } \\
(n=11)\end{array}$ & $\begin{array}{c}\text { CVID } \\
(n=21)\end{array}$ \\
\hline Lymphocytes [\% (cells/ $\mu \mathrm{l})$ ] & 44 (3094) & $40(2085)$ \\
\hline CD3 [\% (cells/ $\mu \mathrm{l})]$ & $88^{*}\left(2937^{*}\right)$ & $76^{*}\left(1676^{*}\right)$ \\
\hline CD4 [\% (cells/ $\mu \mathrm{l})]$ & $46^{*}\left(1730^{*}\right)$ & $39 *\left(836^{*}\right)$ \\
\hline CD8 [\% (cells/ $\mu \mathrm{l})]$ & $30(1036)$ & $29\left(591^{*}\right)$ \\
\hline CD19 [\% (cells/ $\mu \mathrm{l})]$ & $0.45^{*}(15)$ & $15(322)$ \\
\hline 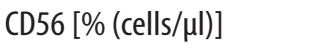 & $9(204)$ & $6\left(158^{*}\right)$ \\
\hline CD4/CD8 ratio & 1.5 & 1.4 \\
\hline
\end{tabular}

$X L A$ - X-linked agammaglobulinaemia, CVID - common variable immunodeficiency, ${ }^{*}$ Mann-Whitney $U$-test $(p<0.05)$

had splenomegaly, one third had hepatomegaly, and 38\% had lymphadenopathy. Bronchial asthma was observed mostly in CVID (29\% vs. 9\%). Local abscesses or furuncles were seen only in XLA boys. In that group, additional diseases such as haemophilia A or Guillain-Barré syndrome were found.
TABLE 2. Clinical symptoms and complications in X-linked agammaglobulinaemia and common variable immunodeficiency children

\begin{tabular}{|c|c|c|}
\hline Symptom/complication, $n$ (\%) & $\begin{array}{c}\text { XLA } \\
(n=11)\end{array}$ & $\begin{array}{c}\text { CVID } \\
(n=21)\end{array}$ \\
\hline \multicolumn{3}{|l|}{ Respiratory tract infections } \\
\hline Lower respiratory tract & $9(81)$ & $18(86)$ \\
\hline ENT & $4(36)$ & $11(52)$ \\
\hline Sinusitis & $5(45)$ & $10(48)$ \\
\hline \multicolumn{3}{|l|}{ Chronic lung disease } \\
\hline Bronchiectasis & $3(27)$ & $7(33)$ \\
\hline Interstitial pneumonia & - & $2(9.5)$ \\
\hline Pulmonary fibrosis & - & $1(4.8)$ \\
\hline \multicolumn{3}{|l|}{ Granulomas } \\
\hline Lung & - & $3(14)$ \\
\hline Liver & - & $1(4.8)$ \\
\hline \multicolumn{3}{|l|}{ Organomegaly } \\
\hline Splenomegaly & - & $9(42)$ \\
\hline Hepatomegaly & - & $7(33)$ \\
\hline \multicolumn{3}{|l|}{ Cytopenia } \\
\hline Neutropenia & $2(18)$ & $6(29)$ \\
\hline ITP & - & $6(29)$ \\
\hline Anaemia & $1(9)$ & $2(9.5)$ \\
\hline Leukopaenia & - & $6(28)$ \\
\hline Lymphadenopathy & - & $8(38)$ \\
\hline \multicolumn{3}{|l|}{ Gastrointestinal symptoms } \\
\hline Diarrhoea & $5(45)$ & $3(14)$ \\
\hline IBD & $2(18)$ & $1(4.8)$ \\
\hline Malabsorption & $1(9)$ & $2(9.5)$ \\
\hline Arthritis & $2(18)$ & - \\
\hline Meningitis/sepsis & $1(9)$ & $4(19)$ \\
\hline Malignancies & - & - \\
\hline Asthma & $1(9)$ & $6(29)$ \\
\hline Skin infections & $2(18)$ & - \\
\hline \multicolumn{3}{|l|}{ Other } \\
\hline Haemophilia A & $1(9)$ & - \\
\hline Guillain-Barré syndrome & $1(9)$ & - \\
\hline
\end{tabular}

XLA - X-linked agammaglobulinaemia, CVID - common variable immunodeficiency

The phenotypical characteristics of basic lymphocytes subsets (CD3+, CD4+, CD8+, CD56+, and CD19+) are shown in Table 3 . The number and percentage of $B$ cells were extremely low in XLA. The total number of CD3+ cells was higher in XLA than in CVID $(p<0.05)$. Statistically significant reductions were observed within the absolute numbers and percentage of TCD4+ in CVID vs. XLA $(p<0.05)$. 


\section{DISCUSSION}

Primary antibody deficiencies account for approximately $50 \%$ of all PIDs in the Polish registry [19]. Among 1700 PID patients followed up in the largest referral centre, over $50 \%$ were diagnosed with PAD and one third required RIgT. We present the demographic and clinical data of 11 boys with XLA and 22 children with CVID diagnosed and treated in one centre.

The median age of disease onset was lower for XLA compared to CVID ( 8.5 months of age vs. 2 years), followed by a shorter diagnostic delay (3 years vs. 9 years). Positive family history and earlier disease manifestation in XLA could result in an earlier diagnosis.

Respiratory tract infections were present in the vast majority of XLA and CVID individuals. No major differences were seen in the frequency of lower respiratory tract infections (LRTI), accounting for approximately 80 $86 \%$ of affected individuals. Similar data were reported in earlier studies [10, 16, 17, 20]. Tavakol et al. [21] indicate high frequency (54.5\%) of ENT infections in all PAD patients, especially in CVID compared to other PAD, which is compatible with our observation.

The gastrointestinal tract is the second organ involved in infections in PAD patients. In large CVID groups, GI infections were reported in $14-22 \%[8,14,15]$. Multiple episodes of gastroenteritis were observed in about $19 \%$ of an Italian XLA cohort [11]. In this study, diarrhoea was more common in XLA than CVID (48\% vs. $14 \%$ ).

Besides infections, autoimmunity, lymphoproliferation, and granulomas are relevant features of immune deficiencies. They are more often observed in CVID than in XLA. Up to $25 \%$ of patients with CVID present various infiltrative complications, usually diagnosed between 20 and 40 years of age. The most common is granulomatous interstitial lung disease and lymphoid interstitial pneumonia $[2,22]$. We observed lung granulomas in three and interstitial pneumonia in two of our patients; however, they were younger. Splenomegaly or hepatosplenomegaly are common in CVID individuals. The relation to some subtypes of CVID classification is considered $[6,7,18,23]$. In our material, approximately $40 \%$ of patients presented splenomegaly and lymphadenopathy, while every third - hepatomegaly. In one CVID boy liver granuloma was recognised. No XLA males had such abnormalities.

About $20-32 \%$ of CVID individuals develop gastrointestinal disorders not related to infections, such as Crohn's disease, Crohn's-like disease, another inflammatory bowel disease, or celiac disease [3]. Some authors regard Crohn's disease to be more connected with XLA [17]. In our study, IBD was diagnosed in two males with XLA (18\%) and one girl with CVID (5\%), as reported earlier [24].

Mechanisms of autoimmune-associated disorders in CVID and XLA are not clear and include many theories from impaired clearance of apoptotic cells to diminished tolerance and dysregulated T-cell functions [25, 26]. In CVID autoimmune thrombocytopaenia and autoimmune haemolytic anaemia are considered the most frequently observed autoimmune phenomena, with a rate of $5-8 \%$. Moreover, this can precede the diagnosis of CVID in some individuals. XLA is considered to have a low risk of autoimmunity, but some data indicate higher than expected the incidence of anaemia, thrombocytopaenia, and neutropaenia [23, 27, 28]. Our own observations showed a higher prevalence of autoimmune cytopaenia in CVID compared to XLA patients. The broad spectrum of clinical symptoms in CVID patients presented by Grześk et al. is compatible with our observations [29].

The real incidence of malignancies in XLA is not known. The CVID group is regarded to be at higher risk of developing a malignancy. Lymphoid malignancies are more prevalent in younger patients, while adults are more susceptible to gastrointestinal tract malignancies $[6,17]$. None of our patients developed a malignancy. This may be connected with the younger age of observed patients and can still be diagnosed in adults.

We noticed a statistically lower percentage and absolute count number of B cells in XLA, while in CVID - a lower absolute number of TCD3+ and CD4+ lymphocytes $(p<0.05)$.

\section{CONCLUSIONS}

Recurrent infections are the most prevalent features of XLA and CVID and physicians should be aware of such rare diseases. Early onset of infections in males favours the diagnosis of XLA, but further diagnostic procedures should be recommended. Non-infectious complications including hypertrophy of lymphoid tissue and autoimmune cytopaenia are more suggestive of CVID. Greater awareness of paediatricians and general physicians leads to proper and earlier diagnosis, easier treatment, and avoidance of severe life-threatening complications.

\section{DISCLOSURE}

The authors declare no conflict of interest.

\section{REFERENCES}

1. Reust CE. Evaluation of Primary Immunodeficiency Disease in Children. Am Fam Physician 2013; 87: 773-778.

2. Webster ADB. Clinical and immunological spectrum of common variable immunodeficiency (CVID). Iran J Allergy Asthma Immunol 2004; 3: 103-113.

3. Kumar Y, Bhatia A. Common variable immunodeficiency in adults: current diagnostic protocol and laboratory measures. Expert Rev Clin Immunol 2013; 9: 959-977.

4. Piqueras B, Lavenu-Bombled C, Galicier L, et al. Common variable immunodeficiency patient classification based on impaired B cell memory differentiation correlates with clinical aspects. J Clin Immunol 2003; 23: 385-400. 
5. Warnatz K, Denz A, Drager R, et al. Severe deficiency of switched memory B cells (CD27(1) $\operatorname{IgM}(2) \operatorname{IgD}(2))$ in subgroups of patients with common variable immunodeficiency: a new approach to classify a heterogenous disease. Blood 2002; 99: 1544-1551.

6. Wehr C, Kivioja T, Schmitt C, et al. The EUROClass trial: defining subgroups in common variable immunodeficiency. Blood 2008; 111: $77-85$

7. Jolles $\mathrm{S}$. The variable in common variable immunodeficiency: a disease of complex phenotypes. J Allergy Clin Immunol Pract 2013; 1: 545-556.

8. Quinti I, Soresina A, Spadaro G, et al. Long-term follow-up and outcome of a large cohort of patients with common variable immunodeficiency. J Clin Immunol 2007; 27: 308-316.

9. Bruton O. Agammaglobulinemia. Pediatrics 1952; 9: 722-728.

10. Pac M, Mikoluc B, Pietrucha B, et al. Clinical and immunological analysis of patients with X-linked agammaglobulinemia - single center experience. Centr Eur J Immunol 2013; 38: 367-371.

11. Plebani A, Soresina A, Rondelli R, et al. Clinical, Immunological, and Molecular Analysis in a Large Cohort of Patients with X-linked Agammaglobulinaemia: An Italian Multicenter Study. Clin Immunol 2002; 104: 221-230.

12. Abolhassani H, Sagvand TB, Shokuhfar T, et al. A review on guidelines for management and treatment of common variable immunodeficiency. Exp Rev Clin Immunol 2013; 9: 561-574.

13. Bonilla FA, Barlan I, Chapel H, et al. International consensus document (ICON): common variable immunodeficiency. J Allergy Clin Immunol Pract 2016; 4: 38-57.

14. Gathmann B, Mahlaoui N, Gerard L, et al. Clinical picture and treatment of 2212 patients with common variable immunodeficiency. J Allergy Clin Immunol 2014; 134: 116-126.

15. Mohammadinejad P, Aghamohammadi A, Abolhassani H, et al. Pediatric patients with common variable immunodeficiency: longterm follow-up. J Invest Allergol Clin Immunol 2012; 22: 208-214.

16. Toth B, Volokha A, Mihas A, et al. Genetic and demographic features of X-linked agammaglobulinemia in Eastern and Central Europe: A cohort study. Mol Immunol 2009; 46: 2140-2146.

17. Park MA, Li JT, Hagan JB. Common variable immunodeficiency: a new look at an old disease. Lancet 2008; 372: 489-502.

18. Piatosa B, Pac M, Siewiera K, et al. Common variable immune deficiency in children - clinical characteristics depending on defect in peripheral B cell maturation. J Clin Immunol 2013; 33: 731-741.

19. Pac M, Bernatowska E. Comprehensive activities to increase recognition of primary immunodeficiency and access to immunoglobulin replacement therapy in Poland. Eur J Pediatr 2016; 175: 1099-1105.

20. Hernandez-Trujillo V, Scalchunes C, Cunningham-Rundles C, et al. Autoimmunity and inflammation in X-linked agammaglobulinaemia. J Clin Immunol 2014; 34: 627-632.

21. Tavakol M, Kouhi A, Abolhassani H, et al. Otological findings in pediatric patients with hypogammaglobulinemia. Iran J Allergy Asthma Immunol 2014; 13: 166-173.

22. Aghamohammadi A, Allahverdi A, Abolhassani H, et al. Comparison of pulmonary diseases in common variable immunodeficiency and X-linked agammaglobulinaemia. Respirology 2009; 15: 289-295.

23. Abolhassani H, Amirkhasani D, Parvaneh N, et al. Autoimmune phenotype in patients with common variable immunodeficiency. J Investig Allergol Clin Immunol 2013; 23: 323-329.

24. Pac M, Bernatowska E, Kierkus J, et al. Gastrointestinal disorders next to respiratory infections as leading symptoms of X-linked agammaglobulinaemia in children - 34-year experience of a single center. Arch Med Sci 2017; 13: 412-417.

25. Boileau J, Mouillot G, Gerard L, et al. Autoimmunity in common variable immunodeficiency: correlation with lymphocyte phenotype in the French DEFI study. J Autoimm 2011; 36: 25-32.
26. Seidel MG. Autoimmune and other cytopenias in primary immunodeficiencies: patomechanism, novel differential diagnoses, and treatment. Blood 2014; 124: 2337-2344.

27. Brandt D, Gershwin ME. Common variable immune deficiency and autoimmunity. Autoimm Rev 206; 5: 465-470.

28. Notarangelo LD. Primary immunodeficiencies (PIDs) presenting with cytopenias. Hematology 2009; 139-143.

29. Grześk E, Dąbrowska A, Urbańczyk A, et al. Pospolity zmienny niedobór odporności - choroba o wielu obliczach. Pediatr Pol 2018; 93: 186-191. 